\title{
A Fully Integrated Conductive and Dielectric Additive Manufacturing Technology for Microwave Circuits and Antennas
}

\author{
Mengze Li $^{\# 1}$, Yang Yang ${ }^{\# 2}$, Yunpeng Zhang ${ }^{\$ 3}$, Francesca Iacopi ${ }^{\# 4}$, Shlomit Ram ${ }^{* 5}$, Jaim Nulman*6 \\ ${ }^{\#}$ Faculty of Engineering and Information Technology, University of Technology Sydney, Australia \\ ${ }^{\$}$ School of Electronic Science and Engineering, University of Electronic Science and Technology of China, China \\ *Nano Dimension, Ness Ziona, Israel \\ 1'mengze.li@student.uts.edu.au, \{2yang.yang-1, ${ }^{4}$ francesca.iacopi $\} @ u t s . e d u . a u,{ }^{3}$ zyp_ee@163.com, $\left\{{ }^{5}\right.$ shlomit.r, ${ }^{6}$ jaim $\} @$ nano-
} di.com

\begin{abstract}
A fully-integrated additive manufacturing (AM) approach for microwave devices is presented in this work. The applied AM technology can print the circuit models simultaneously with conductive and dielectric materials. Taking advantage of this one-stop 3D manufacturing technology, multilayer circuit board with vias or holes can be prototyped rapidly and precisely. The electrical properties of the dielectric ink material are measured up to $40 \mathrm{GHz}$ using a quasi-optical cavity test system. To further demonstrate the merit of this technology, an infilled-ground transmission line, as well as a microstrip patch antenna, are designed and fabricated. For proof-of-concept, the return loss, gain and radiation patterns of the antenna are measured, which demonstrate a good agreement with the simulated results.
\end{abstract}

Keywords - Additive manufacturing, 3D printing technology, infilled-ground, transmission line.

\section{INTRODUCTION}

3D printing technology, firstly emerged in the 1980s, opened a revolutionary era of manufacturing. As an emerging technology, 3D printing is vibrantly redefining manufacturing concepts and their capabilities. In the early days, 3D printing technology was regarded suitable only for the production of mechanical parts and aesthetic prototypes, such as work tools and statues, due to the limitation of available printing materials and fabrication accuracy. In recent times, additive manufacturing (AM) capabilities have extended to cover an increasing spectrum of materials, along with an increased improvement of precision machining, repeatability and reliability. Taking advantage of a broad range of additive manufacturing materials and printing approaches, microwave electronic circuits have been widely achieved, for example of waveguides [1-2], frequency selective surface [3], antennas[4] and transmission lines [5]. It is noted that most of these works employ a single-material 3D printer, which is fairly common in industry. Often, such prototypes were printed in either pure metallic material or pure dielectric material. However, many microwave circuits need both conductive and dielectric materials. Therefore, there is an urgent need for an integrated conductor and dielectric printing technique. In [4], to construct an inkjet-printed patch antenna, silver ink was manually

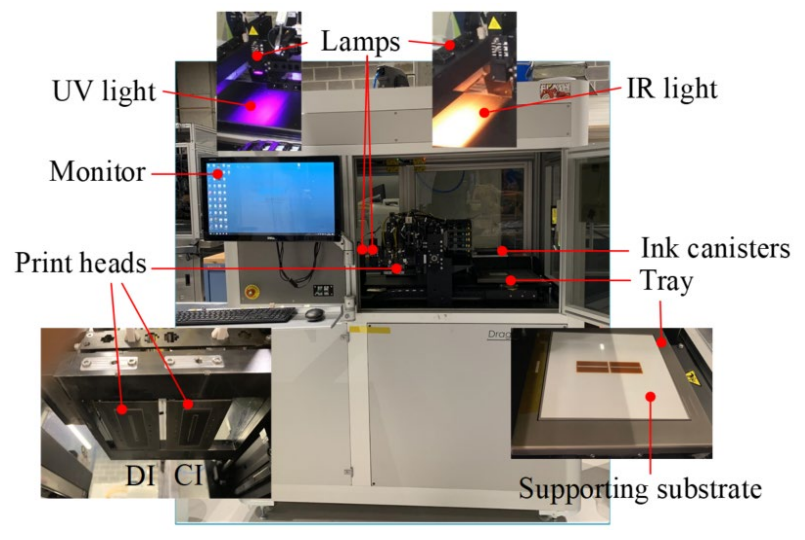

Fig. 1 Composition of the one-stop 3D printing system.

adhered to a prepared paper substrate by a metallic 3D printer. A multi-material 3D printing approach with high accuracy was proposed in [5]. However, the printing time was significantly long due to the adopted solution of aerosol jet printing.

In this paper, microwave transmission lines and microstrip patch antenna designs are implemented using a commercial additive manufacturing system, which dispenses both conductive and dielectric materials enabling the formation of intercalated layers and resulting in completely planar surfaces. The circuit models configured with both conductive and dielectric materials that can be fabricated in a single process rapidly and at low cost. The complete printing process can be carried out at normal temperature and standard atmosphere pressure. As shown in Fig.1, the printer dimensions are $810 \times 1180 \times 2000(\mathrm{~mm})$. Specially, the manufacturing system has two printing heads with 512 nozzles each. Silver nanoparticle ink and ultraviolet (UV) curable acrylates ink can be printed simultaneously to a supporting substrate from these nozzles. On each layer, dielectric and conductive inks are jetted on the substrate, where UV light and infrared radiation (IR) lamps are turned on to solid the inks, respectively. Once the printing process is completed, the samples can be easily removed from the supporting substrate. In addition, multiple prototype samples can be produced at one time. 


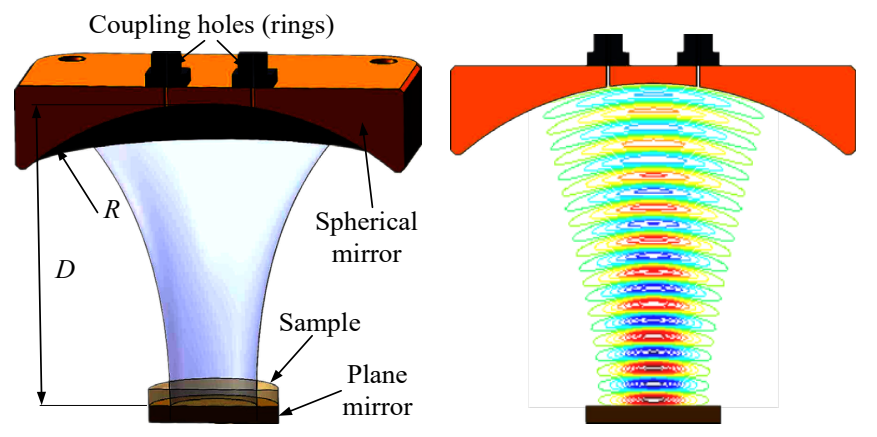

(a)

Fig. 2 The applied semi-symmetric quasi-optical cavity measurement setup: (a) Schematic diagram, and (b) Fundamental mode field distribution.

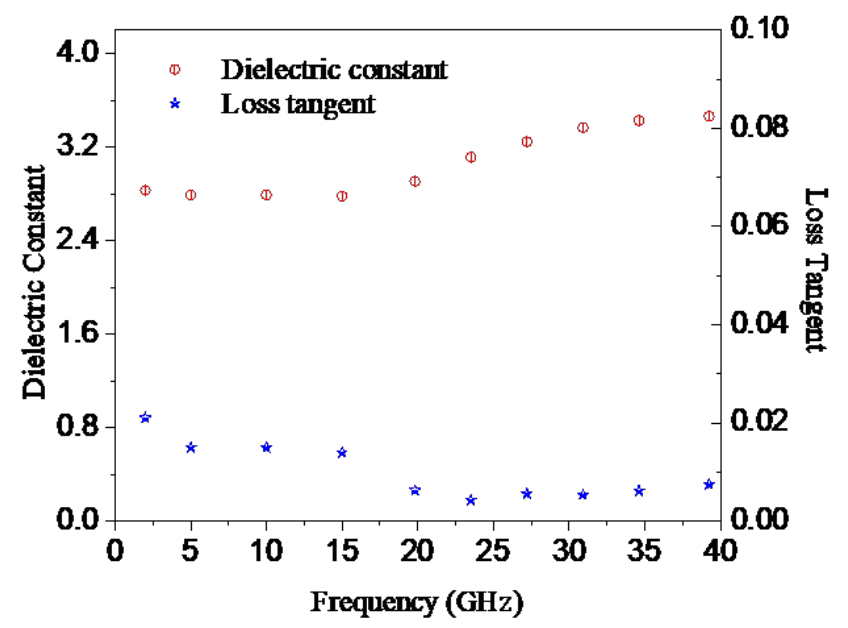

Fig. 3. Acrylates dielectric constant and loss tangent response.

To characterize the dielectric materials, a quasi-optical cavity test system is utilized to measure the electrical properties of the acrylates up to $40 \mathrm{GHz}$. Transmission lines with embedded top metal line are designed and analyzed to test the fabrication of multilayer circuits. The signal propagation performance with different percentages of infilled-ground in a transmission line model is also demonstrated. Finally, a patch antenna is designed and fabricated to verify the process and prototype modeling. Good agreement between the measured and simulated results is achieved.

\section{MATERIALS AND AdDITIVE MANUFACTURING}

The dielectric properties of materials play an important role in the design of $3 \mathrm{D}$ printed microwave circuits. As characterized by DAK-TL3.5-P from $200 \mathrm{MHz}$ to $20 \mathrm{GHz}$, the dielectric ink 1086A, whose main ingredients is acrylates, exhibits a dielectric constant of 2.7-2.9 and the loss tangent less than 0.029 (with a sample size of $30 \mathrm{~mm}$ ). To characterize the material performance above $20 \mathrm{GHz}$, quasi-optical cavity approach, as shown in Fig. 2, is adopted. Similar to the approach demonstrated in [6], samples of acrylates are characterized by cavity resonant method with the highest possible accuracy. Fig. 2(a) is the schematic diagram of a semisymmetric quasi-optical cavity composed of a spherical mirror and a plane mirror. The plane mirror is usually a metal circular

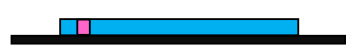

(a)

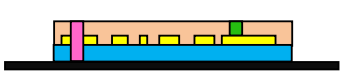

(c)

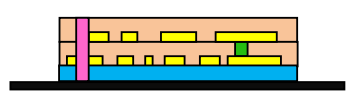

(e)

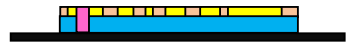

(b)

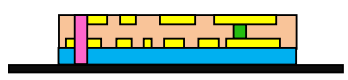

(d)

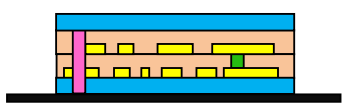

(f)

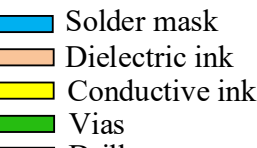

(1)

Fig. 4. Steps of the conductive and dielectric one-stop 3D printing fabrication.

platform, which can move along the axis for adjusting the cavity length $D$. The spherical mirror has a curvature radius of $R$. Two coupling holes (or rings) connected with the vector network analyzer (VNA) are symmetrically located in the middle of the mirror. The size of the quasi-optical cavity is much larger than the working wavelength. Under the paraxial approximation, the field distribution in the cavity exists in the form of the Gaussian beam, which is the quasi-TEM plq $_{\text {wave. Among the different }}$ modes, fundamental mode $\mathrm{TEM}_{00 q}$ is set as the working mode due to its concentrated electromagnetic energy distribution, as shown in Fig. 2 (b).

Finally, the material sample placed in the holder has the same diameter with the plane mirror, whose thickness depends on the sample loss and test frequency. Fig. 3 illustrates the measured dielectric constant and loss tangent of the acrylates dielectric material. Due to the limitation of the cavity size used in the measurement, the experiment was only carried out from 18 to $40 \mathrm{GHz}$. Combined with the data characterized by DAKTL3.5-P, Fig. 3 gives the radio frequency (RF) characteristics of the dielectric ink 1086A, from $200 \mathrm{MHz}$ to $40 \mathrm{GHz}$. According to Fig. 3, the dielectric constant develops gradually from 2.8 to 3.4 , while the loss tangent varies from $0.016-0.024$ during 1-20 GHz, and keeps lower than 0.008 during 20-40 $\mathrm{GHz}$.

Different from the traditional material jetting technologies, the one-stop additive manufacturing system uses the piezoelectric based nozzle with deposition in an ink-filled chamber behind each nozzle instead of a heating element. Fig. 4 demonstrates the printing steps of the conductive and dielectric one-stop additive manufacturing system. Soldering masks are usually printed on the bottom and top of the prototypes for connections to other devices or lumped components. The soldering masks consist of the same dielectric inks as used in the manufactured device. During the printing process, UV and IR lamps are utilized after each layer is printed, to solid polymer and the silver inks, respectively. Multiple metallic layers with vias and through holes/plated through holes 

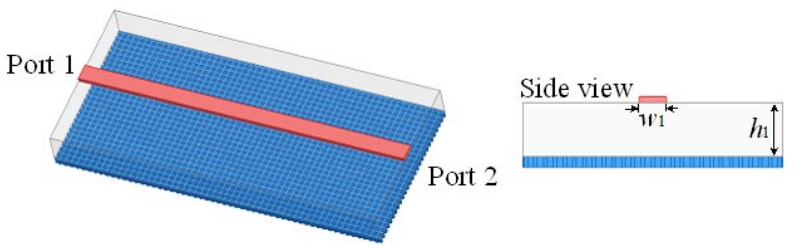

(a)

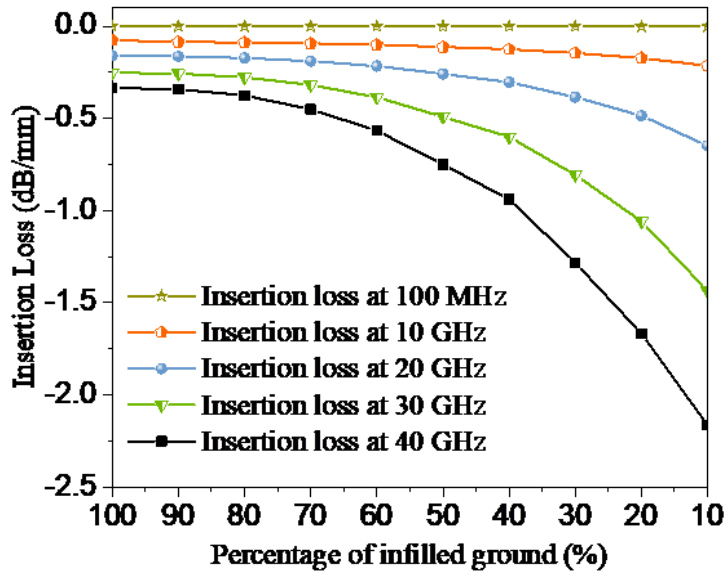

(b)

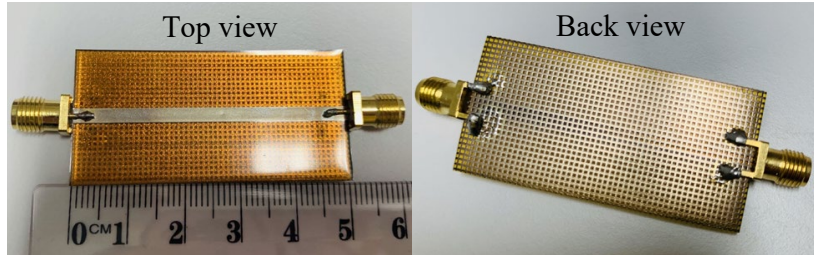

(c)

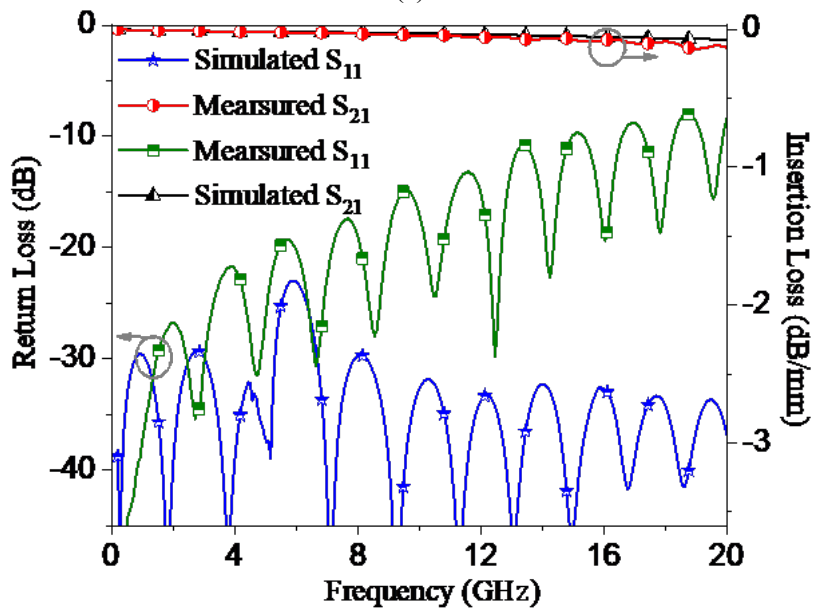

(d)

Fig. 5. (a) Construction of infilled-ground transmission line. (b) Simulated insertion loss varied percentage of infilled-ground at different frequency. (c) Photograph of the infilled-ground transmission line model and its measured results of insertion and return loss. ( $w_{1}=2.6 \mathrm{~mm}, h_{1}=1 \mathrm{~mm}$.)

can be achieved as long as the design rule is followed. The fabrication accuracy can reach to $125 \mu \mathrm{m}$ in traces and $125 \mu \mathrm{m}$ in spaces, while the minimum layer thicknesses are $0.3 \mu \mathrm{m}$ for conductive layer and $2.5 \mu \mathrm{m}$ for dielectric layer.

\section{INVESTIGATION OF INFILLED-GROUND TRANSMISSION} LINE

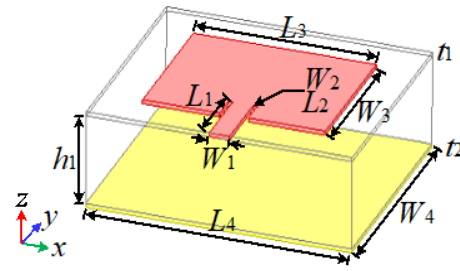

(a)

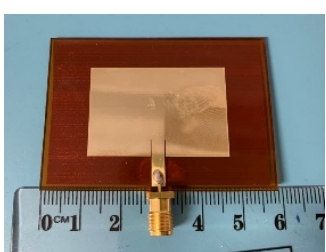

(b)

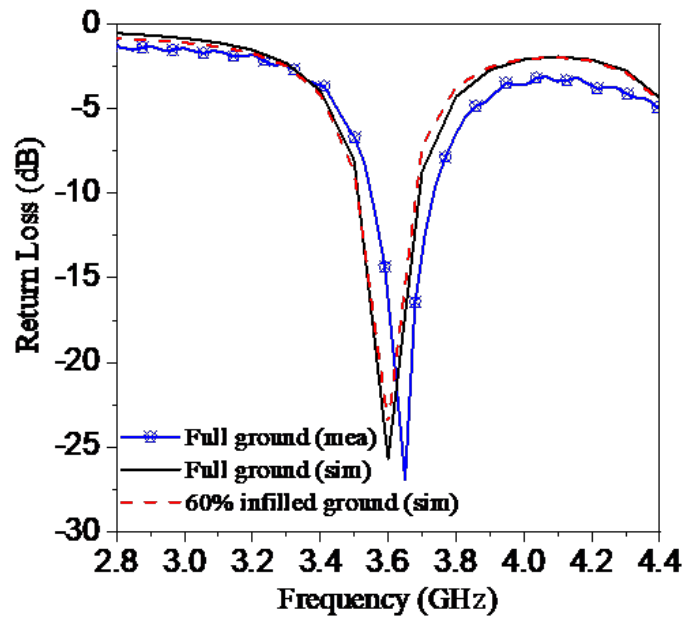

(c)

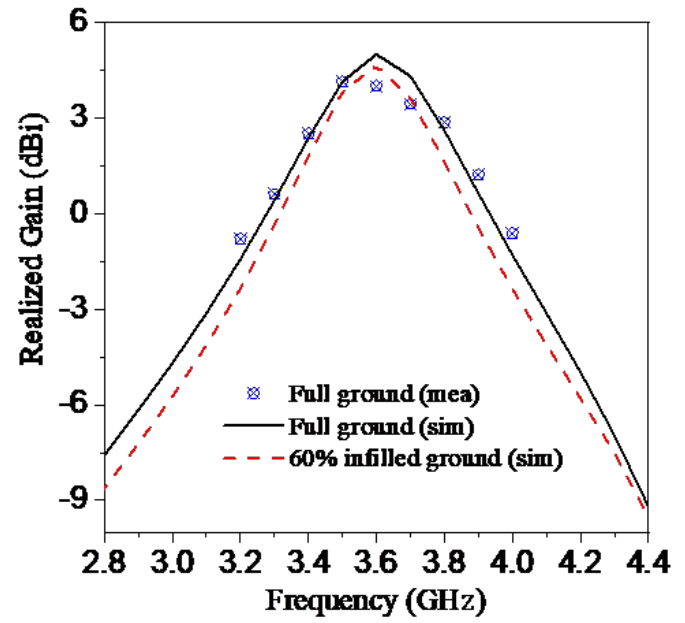

(d)

Fig. 6. Antenna prototype: (a) Design model in HFSS, (b) Photograph of full ground patch antenna, (c) Simulated and measured return loss, and (d) Simulated and measured realized gain (including a simulated $60 \%$ infilled ground patch antenna). Dimensions: $t_{1}=t_{2}=0.02, h_{1}=1.46, L_{1}=11, L_{2}=4, L_{3}=37$, $L_{4}=57, W_{1}=3.3, W_{2}=0.3, W_{3}=22.9, W_{4}=36.9$, (unit: $\mathrm{mm}$ ).

In additive manufacturing, the cost of the fabrication is mainly dictated by the consumption of the materials. Replacing a full metallic ground with an infilled one is an appropriate way to reduce the consumption of the conductive ink. Therefore, an infilled-ground transmission line is designed, fabricated and measured. Fig. 5(a) depicts the structure of the transmission line with infilled-ground plane at the bottom. The total dimension of the transmission line is $24 \mathrm{~mm} \times 50 \mathrm{~mm} \times 1 \mathrm{~mm}$ with a percentage of infilled-ground. Thickness of each silver layer was set to $20 \mu \mathrm{m}$. As shown in Fig. 5(b), with the decrease of 


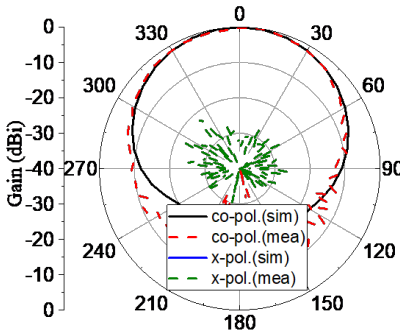

(a)

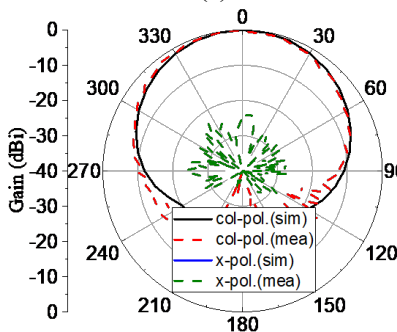

(c)

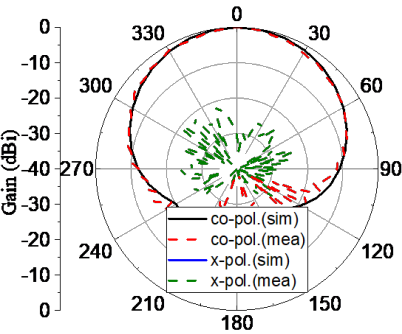

(e)

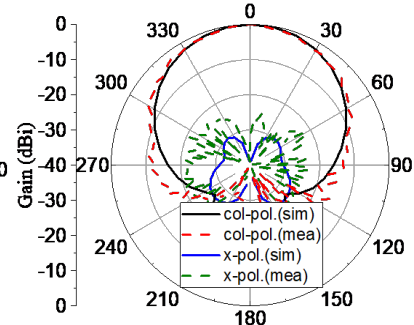

(b)
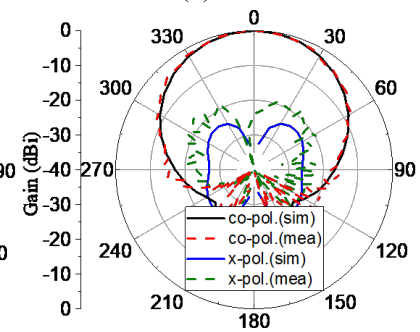

(d)

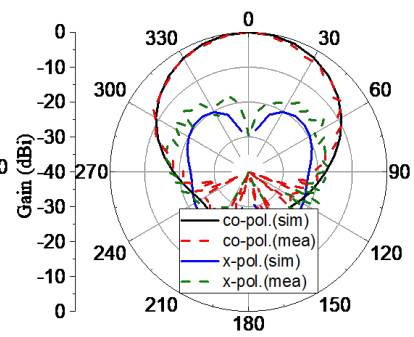

(f)
Fig. 7. Radiation patterns of the prototyped full-ground patch antenna. (a) $Y O Z$-plane at $3.5 \mathrm{GHz}$, (b) $X O Z$-plane at $3.5 \mathrm{GHz}$, (c) $Y O Z$-plane at $3.6 \mathrm{GHz}$, (d) XOZ-plane at $3.6 \mathrm{GHz}$, (e) $Y O Z$-plane at $3.7 \mathrm{GHz}$, (f) $X O Z$-plane at $3.7 \mathrm{GHz}$

percentage of infilled-ground, the insertion loss increases gradually versus frequency. Furthermore, with the increase of frequency, the insertion loss resulted by the infilled-ground becomes greater. Therefore, a trade-off between the tolerance of insertion loss and the percentage of infilled-ground should be considered in the design. Finally, the transmission lines are fabricated. Fig. 5(c) shows the photographs of the top view and back view of the infilled-ground transmission line. The simulated and measured results are illustrated in Fig. 5(d). Good agreement can be achieved between the simulated and measured insertion loss. However, the simulated and measured return loss has some discrepancies, which may be resulted from the connector issues, which can be improved by using the highend SMA connectors with a stable performance up to $20 \mathrm{GHz}$.

\section{Microstrip PATCh ANTENNA DESIGN}

To further validate the design and fabrication approach, a patch antenna operating at $3.6 \mathrm{GHz}$ is designed using EM software HFSS. Fig. 6(a) demonstrates the construction of the patch antenna and its dimension parameters. Fig. 6(b) is the photograph of the fabricated full ground patch antenna. When the full ground of this patch antenna is replaced by $60 \%$ infilledground, as shown in Fig. 6(c), the central frequency and operational frequency band almost keep constant. In addition, the realized gain of the patch antenna with full ground and $60 \%$ infilled-ground are shown in Fig. 6(d). The peak value of the realized gain decreases from 4.97 to $4.68 \mathrm{dBi}$, when the full ground changes to $60 \%$ infilled-ground. The measured insertion loss and realized gain are illustrated in Figs. 6(c) and(d), respectively, which verify a good agreement with the simulated ones. Finally, as shown in Fig. 7, the simulated and measured radiation patterns of a full-ground patch antenna are presented at 3.5, 3.6 and $3.7 \mathrm{GHz}$ in the YOZ-plane and XOZ-plane, respectively. Good agreements are achieved between the simulated and the measured results.

\section{CONCLUSION}

In this paper, a novel conductor and dielectric one-stop additive manufacturing technology has been introduced. The $\mathrm{RF}$ characteristics of the dielectric material has been characterized up to $40 \mathrm{GHz}$. For further investigations, the infilled-ground transmission line and a patch antenna are designed and fabricated for proof-of-concept. According to the study, the devices with full ground and $60 \%$ infilled ground do not show significant difference in its RF performance, which can be accepted by the industry-tolerance. Finally, the validity of the models are discussed by comparing the simulated and measured results, which suggests the possibility of applying this low-cost and flexible-designing technology into common PCB production.

\section{REFERENCES}

[1] K. Y. Chan, R. Ramer, and R. Sorrentino, "Low-cost ku-band waveguide devices using 3-D printing and liquid metal filling," IEEE Trans. Microw. Theory Techn., vol. 66, no. 9, pp. 3993-4001, Sep. 2018.

[2] M. D'Auria, W. J. Otter, and J. Hazell et al., "3-D printed metal-pipe rectangular waveguides," IEEE Trans. Compon. Packag. Manuf. Technol., vol. 5, no. 9, pp. 1339 - 1349, Sep. 2015.

[3] B. Sanz-Izquierdo, and E. A Parker, "3-D printing of elements in frequency selective arrays," IEEE Trans. Antennas Propag., vol. 62, no. 12, pp. 6060-6066, Dec. 2014.

[4] S. Kim, Y.J. Ren, and H. et al., "Monopole antenna with inkjet-printed EBG array on paper substrate for wearable applications," IEEE Antennas Wirel. Propag. Lett, vol. 11, pp. 663-666, Jun. 2012.

[5] F. Cai, Y. Chang, and K. Wang et al., "Low-loss 3-D multilayer transmission lines and interconnects fabricated by additive manufacturing technologies," IEEE Trans. Microw. Theory Techn., vol. 64, no. 10, pp. 3208-3216, Oct. 2016.

[6] J. Krupka, "Frequency domain complex permittivity measurements at microwave frequencies," Meas. Sci. Technol., vol. 17, no. 6, pp. R55R70, 2006. 